\title{
Evolving concepts and consensus in challenging shoulder problems: a European perspective
}

\author{
Giuseppe Milano ${ }^{1,2}$ [D $\cdot$ Frank Martetschläger ${ }^{3,4} \cdot$ Ladislav Kovačič $^{5}$
}

Received: 12 April 2021 / Accepted: 26 April 2021 / Published online: 15 May 2021

c European Society of Sports Traumatology, Knee Surgery, Arthroscopy (ESSKA) 2021

Acromioclavicular (AC) joint injuries are among the most common sports-related disorders of the shoulder, especially in young men [8], and in recent years scientific interest in them has grown rapidly. Increasing clarification of AC joint anatomy and function has led to the publication of a growing number of surgical techniques designed to address AC joint instability. At a certain point, however, the abundance of options seemed to be creating confusion and undermining efforts to unveil new science-based strategies. Despite the availability of multiple surgical options, there appeared to be doubts and uncertainties on how to correctly manage these injuries. We, therefore, felt that the time was ripe for a structured analysis of the field.

This special issue of KSSTA contains a systematic review of all the available techniques for surgical treatment of acute and chronic $\mathrm{AC}$ joint dislocation [6, 7].

After reviewing more than 150 papers, the authors concluded that biological and synthetic reconstructions are the most suitable options in both acute and chronic settings. An open approach is probably still the most common, even

Giuseppe Milano

giuseppe.milano@unibs.it

Frank Martetschläger

frank.martetschlaeger@atos.de

Ladislav Kovačič

ladislav.kovacic@kclj.si

1 Department of Medical and Surgical Specialties, Radiological Sciences, and Public Health, University of Brescia, Brescia, Italy

2 Department of Bone and Joint Surgery, ASST Spedali Civili, Piazzale Spedali Civili 1, 25123 Brescia, Italy

3 Department for Orthopaedic Sports Medicine, Technical University of Munich, Munich, Germany

4 Center for Shoulder and Elbow Surgery, ATOS Clinic, Effnerstrasse 38, 81925 Munich, Germany

5 University Medical Centre of Ljubljana, Zaloška cesta 2, 1000 Ljubljana, Slovenia though there is certainly a growing interest in arthroscopic AC joint reconstruction techniques among surgeons. Of the various surgical options, anatomical reconstructions showed the best functional performance in both settings.

To avoid overlooking any step in the natural history of treated or untreated conditions of the $\mathrm{AC}$ joint, this special publication carefully considers issues related not only to AC joint instability, but also to osteoarthritis [9].

Nevertheless, systematic literature reviews only represented a solid foundation for a more ambitious plan. In October 2018, in Athens, the European Shoulder Associates (ESA), a special section of the European Society of Sports Traumatology, Knee Surgery and Arthroscopy (ESSKA), held its first Closed Meeting. The focus of this successful event was the diagnosis and treatment of $\mathrm{AC}$ joint disorders. All the members of the international panel of experienced shoulder surgeons attending the meeting took part in the first round of a Consensus Project, which took four more rounds, held in the course of a year, to complete. The last round took place at the ESSKA Specialty Days Meeting in Madrid in November 2019. After carefully considering the experts' opinions and literature findings, and considering in depth all the stages in the diagnostic-treatment algorithm, a final consensus was reached on the main and most controversial issues surrounding $\mathrm{AC}$ joint dislocation.

A detailed description of the ESA-ESSKA Consensus Project on the AC joint and its outcomes can be found in this special issue [4]. In particular, the consensus document clearly states that a true anteroposterior view or a bilateral Zanca radiograph without loading of the arm is sufficient for correct diagnosis and classification of AC joint dislocation. Moreover, the Rockwood classification, as modified by the International Society of Arthroscopy, Knee Surgery and Orthopedic Sports Medicine (ISAKOS) statement, is still considered the most valid. Most important, a clear demarcation line between acute and chronic cases was consensually set at 3 weeks. From a surgical standpoint, anatomical reconstructions were confirmed to be the best option. 
Arthroscopically assisted reconstruction using a suspensory device with no need for further biological augmentation was the strategy recommended for acute injuries, whereas the use of biological reconstruction with tendon graft should be preferred in chronic cases.

Although it can hardly be claimed that all the burning questions around the diagnosis and treatment of AC joint disorders have now been answered, the present special issue is nevertheless well worth reading, as it can be regarded as a landmark review of current knowledge in the field.

Another focus of this special issue is the treatment of rotator cuff tears (RCTs).

Nowadays, RCTs are successfully treated by arthroscopy. The passing years have brought tremendous improvements in surgical techniques, implants, equipment and instruments, as well as surgeons' skills, which together allow optimal visualization of and access to the torn and retracted tendons, and facilitate the treatment even of massive tears.

The value of arthroscopic treatment of massive RCTs is underlined by a systematic review included in this issue [3]. The authors showed that arthroscopic partial repair of massive RCTs can lead to significant improvements in terms of shoulder function and pain relief, and a lower re-tear rate than previously reported. However, it must be underlined that a $36 \%$ failure rate should still be considered too high, and that better definition of the patient cohorts that stand to benefit most from this treatment is mandatory.

Treatment of massive RCTs in the chronic setting and of irreparable tears in the younger and active population is still among the greatest challenges faced by shoulder surgeons. To address these issues, the ESA-ESSKA scientific program of the 2019 ESSKA Speciality Days Meeting in Madrid focused on the treatment of massive and irreparable RCTs (MIRCTs). During the event, the latest trends and most reliable techniques in MIRCT treatment were reported, including techniques to improve tendon-to-bone healing, well-known and modern tendon transfer procedures, superior capsule reconstruction, graft augmentation, and subacromial balloon implantation methods, and reverse shoulder arthroplasty techniques. The key take-home message of the meeting was that, for any reconstructive procedure, the best possible local environment should be created, to enhance biological processes.

Indeed, given the continuous improvements in knowledge of biomechanics and stable cuff anchorage, the weak link in rotator cuff repair procedures is now considered to be not mechanical, but biological. Poor tissue quality and over tensioning due to retraction or poor blood supply at the bone-tendon interface can negatively affect healing potential. In a randomized controlled trial, Ruìz Iban et al. [5] showed that nanofracturing at the footprint reduced re-tear rates by approximately $50 \%$. This should be considered as an easy possible addition to normal footprint preparation in any rotator cuff repair procedure. It remains to be seen whether, in the future, additional steps, such as platelet-rich plasma or stem cell injections, will significantly improve healing and be implemented in daily surgical practice.

Given the scarcity of prospective randomized data comparing different treatments, shoulder surgeons need to carefully ponder the various options for each case and consider patient-specific prognostic factors. Thanks to the ongoing work of many dedicated researchers around the world, not least the ESA-ESSKA members, the options for our patients should become even better in the future. This, after all, is what we are all working for.

Finally, a few articles in this special issue deal with shoulder instability. Despite the availability of an exhaustive body of literature on this topic, the problem of instability management is still debated. The studies published in this special issue tackle all the various questions about indications, surgical techniques, and fixation devices, as well as revision surgery problems $[1,2,10]$. Thus, our knowledge is expanding all the time, albeit sometimes in small steps, and ESA-ESSKA intendeds to contribute to this process with ongoing enthusiasm and commitment.

Anterior shoulder instability has been the chosen topic for many ESA-ESSKA projects in recent years. We held an interesting and successful ESA-ESSKA closed meeting in November 2020, during which the importance of age and time in the management of patients with anterior shoulder instability was extensively debated. Patient age is clearly an important factor in the decision-making algorithm: we all know very well that the therapeutic approach to individuals with the same type of shoulder instability differs greatly in young adults compared with middle aged patients. Time as a further factor influencing our therapeutic approach raises additional dilemmas. Just think how often you have asked yourselves whether it is too late to perform a labral or bony Bankart lesion repair, or to treat a Hill-Sachs defect. In the lack of clear evidence, consensus among dedicated experts could provide valuable guidelines. In addition, this will be the goal of the forthcoming ESA-ESSKA Consensus Project on shoulder instability.

Shoulder instability will also be the topic of the ESAESSKA scientific program at the next 2021 ESSKA Speciality Days Meeting: anterior shoulder instability-diagnosis and treatment. The term "anterior shoulder instability" covers a broad spectrum of clinical and pathological patterns that would be better addressed through a case-based approach. A further aim of the ESA-ESSKA instability project is to provide an important publication-case-based guide (with video) to appropriate treatment, for use in everyday clinical practice. We are confident that this book will find its place in the shoulder surgeon's bookcase. 
Funding None.

\section{Declarations}

Conflict of interest The authors declare that they have no conflict of interest.

Ethical approval This article does not contain any studies with human participants or animals performed by any of the authors.

\section{References}

1. Ali ZS, Hurley ET, Jamal MS, Horan MP, Montgomery C, Pauzenberger L et al (2020) Low rate of recurrent instability following the open Latarjet procedure as a revision procedure for failed prior stabilization surgery. Knee Surg Sports Traumatol Arthrosc. https://doi.org/10.1007/s00167-020-06155-6

2. Dyrna FGE, Ludwig M, Imhoff AB, Martetschläger F (2020) Off-track Hill-Sachs lesions predispose to recurrence after nonoperative management of first-time anterior shoulder dislocations. Knee Surg Sports Traumatol Arthrosc. https://doi.org/10.1007/ s00167-020-06176-1

3. Haleem A, Gohal C, Leroux T, Henry P, Alolabi B, Khan M (2020) Primary arthroscopic repair of massive rotator cuff tears results in significant improvements with low rate of re-tear. Knee Surg Sports Traumatol Arthrosc. https://doi.org/10.1007/ s00167-020-06190-3

4. Rosso C, Martetschläger F, Saccomanno MF, Voss A, Lacheta L, ESA DELPHI Consensus Panel (2020) High degree of consensus achieved regarding diagnosis and treatment of acromioclavicular joint instability among ESA-ESSKA members. Knee Surg Sports Traumatol Arthrosc. https://doi.org/10.1007/s00167-020-06286-w

5. Ruiz Ibán MA, Sanchez Alepuz E, Diaz Heredia J, Hachem A-I, Ezagüi Bentolila L, Calvo A et al (2020) Footprint preparation with nanofractures in a supraspinatus repair cuts in half the retear rate at 1-year follow-up. A randomized controlled trial. Knee Surg Sports Traumatol Arthrosc. https://doi.org/10.1007/ s00167-020-06073-7

6. Saccomanno MF, Sircana G, Cardona V, Vismara V, Scaini A, Salvi AG et al (2020) Biologic and synthetic ligament reconstructions achieve better functional scores compared to osteosynthesis in the treatment of acute acromioclavicular joint dislocation. Knee Surg Sports Traumatol Arthrosc. https://doi.org/10.1007/ s00167-020-06217-9

7. Sircana G, Saccomanno MF, Mocini F, Campana V, Messinese $\mathrm{P}$, Monteleone A et al (2020) Anatomic reconstruction of the acromioclavicular joint provides the best functional outcomes in the treatment of chronic instability. Knee Surg Sports Traumatol Arthrosc. https://doi.org/10.1007/s00167-020-06059-5

8. Skjaker SA, Enger M, Engebretsen L, Brox JI, Bøe B (2020) Young men in sports are at highest risk of acromioclavicular joint injuries: a prospective cohort study. Knee Surg Sports Traumatol Arthrosc. https://doi.org/10.1007/s00167-020-05958-x

9. Soler F, Mocini F, Djemeto DT, Cattaneo S, Saccomanno MF, Milano G (2021) No differences between conservative and surgical management of acromioclavicular joint osteoarthritis: a scoping review. Knee Surg Sports Traumatol Arthrosc. https://doi.org/ 10.1007/s00167-020-06377-8

10. Wu IT, Desai VS, Mangold DR, Camp CL, Barlow JD, SanchezSotelo J et al (2020) Comparable clinical outcomes using knotless and knot-tying anchors for arthroscopic capsulolabral repair in recurrent anterior glenohumeral instability at mean 5-year follow-up. Knee Surg Sports Traumatol Arthrosc. https://doi.org/ 10.1007/s00167-020-06057-7

Publisher's Note Springer Nature remains neutral with regard to jurisdictional claims in published maps and institutional affiliations. 